\title{
Odd Couple? Reenvisioning the Relation Between Science and Practice in the Dissemination- Implementation Era
}

\section{Citation}

Weisz, J. R., M. Y. Ng, and S. K. Bearman. 2013. “Odd Couple? Reenvisioning the Relation Between Science and Practice in the Dissemination-Implementation Era." Clinical Psychological Science 2 (1) (December 11): 58-74. doi:10.1177/2167702613501307.

\section{Published Version}

doi:10.1177/2167702613501307

\section{Permanent link}

http://nrs.harvard.edu/urn-3:HUL.InstRepos:16922667

\section{Terms of Use}

This article was downloaded from Harvard University's DASH repository, and is made available under the terms and conditions applicable to Open Access Policy Articles, as set forth at http:// nrs.harvard.edu/urn-3:HUL.InstRepos:dash.current.terms-of-use\#OAP

\section{Share Your Story}

The Harvard community has made this article openly available.

Please share how this access benefits you. Submit a story.

\section{Accessibility}




\title{
Cute Couple or Strange Bedfellows?
}

Re-envisioning the Science-Practice Relationship in Treatment Dissemination-Implementation

\author{
John R. Weisz and Mei Yi Ng \\ Harvard University \\ Sarah Kate Bearman \\ Yeshiva University
}

Running head: Science and Practice of Dissemination and Implementation

Word count (front matter, Abstract, text, Author Notes, and references): 13.846

Submitted: March 14, 2013 


\begin{abstract}
Decades of clinical psychological science have produced many evidence-based interventions that are now undergoing dissemination and implementation (DI), but with little guidance from a DI science that is just now taking shape. Charting a future for DI science and practice, and their complex relationship, will be complicated by significant challenges-the implementation cliff (treatment benefit drops when tested practices are taken to scale), low relevance of most clinical research to actual practice, and differing timetables and goals for DI practice versus research. To address the challenges, and prepare the next generation of psychologists, we propose: making intervention research look more like practice, solving the "too many EBPs” problem, addressing mismatches between interventions and their users, broadening our range of intervention delivery systems, sharpening outcome monitoring and feedback, incentivizing high-risk/high-gain innovations, designing new professional tracks for DI science, and synchronizing and linking the often-insular practice and science of DI.
\end{abstract}

Keywords: dissemination, implementation, evidence-based practice 
An emerging challenge in clinical psychological science is the tension between rigorous testing of interventions_-an ongoing task that is never really finished_and deploying those interventions within clinical practice settings. Moving too quickly from science to practice can make us marketers with products not yet ready for prime time. Moving too slowly can mean lost opportunities to take our work to scale and improve clinical care for those who need it. In this paper we focus on the tension between science and practice-whether the two are a cute couple or strange bedfellows - in the development and diffusion of interventions, and implications of the science-practice relationship for clinical training.

\section{Welcome to COI World.}

This science-practice tension is highlighted by the annual conflict-of-interest (COI) reports that are now routine for faculty in North American universities. In decades past, intervention science and practice were indeed a cute couple (with rather socialist leanings) in which scientists developed and tested treatment protocols and shared these openly for use by practitioners who wanted them. That openness has now given way to a more capitalist model in which these products are "IP,” owned and marketed, often for income beyond the wildest dreams of prior generations in our field. Under these circumstances, a fair study with adverse findings regarding a treatment program can have major consequences-including loss of employment for those in the business of spreading the treatment, loss of income for the IP-holder and partners, and loss of image in the marketplace with concomitant loss of market share. The resulting pressure on scientists who develop marketable products can be significant, and the potential for conflict of interest is real-hence the need for COI reports. While this situation is not new in many fields, it is relatively new for psychological science; this may be a good time for careful thought about the science-practice relationship within our discipline. Can the science of 
developing and testing interventions fairly be done by the same people who are promoting their spread, or do we need strategies for separating the two functions to preserve scientific integrity? What does the answer to this question suggest about how we train the next generation in our field? These questions have become salient in part because of a series of events in science, practice, and public policy, over the past 15 years, including....

- The remarkable growth of the randomized controlled trial (RCT) database encompassing diverse mental health problems across diverse age groups (see e.g., Nathan \& Gorman, 2007; Weisz \& Kazdin, 2010; http://www.cochrane.org/).

- The creation of standards, guidelines, and ultimately criteria to guide the field in identifying interventions that are "empirically supported,” "probably efficacious,” "well-established,” and more generally evidence-based, and reports identifying interventions that meet those criteria (see e.g., Chambless et al., 1998; Silverman \& Hinshaw, 2008).

- Numerous reports and initiatives by government entities, panels, and advocacy groups stressing the importance of using evidence-based practices (EBPs) in mental health care (see, e.g., NAMI, 2003; National Advisory Mental Health Council, 2001).

- An array of initiatives by various state governments, US federal entities, and governments outside the US promoting the identification and increased use of interventions supported by evidence (see e.g., McHugh \& Barlow, 2010).

- The expansion of calls for a broader array of methods for extending the products of intervention research to those who need them, using, for example, advances in technology, nontraditional providers, self-help programs, media outreach, and methods modeled on public health campaigns (see, e.g., Kazdin \& Blase, 2011; Sanders, 2012). 
- The emergence of companies devoted to marketing and spreading treatment materials, trainings, consultations, and sometimes assessments of treatment fidelity and outcome. Often these entities include individuals who are concurrently producing evidence bearing on the products being marketed.

\section{Dissemination and Implementation, Practice and Science}

These developments, taken together, have fueled the spread of tested interventions far beyond research labs, with concomitant attention to the tasks of dissemination and implementation (DI). Dissemination has been defined (NIMH, 2009) as "the targeted distribution of information and intervention materials to a specific public health or clinical practice audience. The intent is to spread knowledge and the associated evidence-based interventions.” Implementation has been defined (NIMH, 2009) as "the use of strategies to adopt and integrate evidence-based health interventions and change practice patterns within specific settings.” Carrying out these two processes is dissemination and implementation practice (DIP). The study of methods for doing these things, and the effects of those methods, provides much of the content of dissemination and implementation science (DIS), a relatively new field of study as applied to EBPs. A case can be made that DIP and DIS should be a part of the training agenda for psychologists, because both are increasingly central to our discipline and because we have so much to learn about which DI practices are effective with which interventions in which contexts. Expanding our agenda in this way will require addressing at least four challenges.

\section{Challenges for DIP and DIS}

I. The implementation cliff. Current evidence suggests that we have a lot to learn about the best ways to disseminate and implement new interventions. One significant challenge is what might be called the implementation cliff, a drop in benefit that often occurs when treatments 
leave the secure base of their university or laboratory settings. Meta-analyses point to a substantial loss in effect size when interventions move from research to practice contexts and when EBPs are tested against usual clinical care (e.g., Wampold et al., 2011; Weisz, JensenDoss, \& Hawley, 2006). In fact, in one recent meta-analysis EBPs did not significantly outperform usual care among studies using (a) clinically referred youths, or (b) youths impaired enough to meet criteria for a formal diagnosis. A relevant example of the drop-off phenomenon is provided by a meta-analysis of Multisystemic Therapy (MST). Although MST research has been exemplary in prioritizing external validity, a meta-analysis of 11 MST studies found that as study designs moved increasingly away from developer control (e.g., graduate student therapists employed and supervised by the treatment developers) and toward conditions of greater external validity (e.g., local therapists and supervisors), effect sizes dropped markedly ( $d=.81$ vs. .26; Curtis, Ronan, \& Borduin, 2004). Similarly, when Addis et al. (2004) compared cognitive behavioral therapy (CBT) for Panic Disorder to treatment as usual, groups did not differ on rates of clinically significant change . There are some apparent exceptions to the drop-off phenomenon (e.g., Foa et al., 2005), but in general interventions appear to suffer both "program drift” and “voltage drop” (personal communication, J. Sherrill) with successive generations beyond the developer’s original implementation (McGrew, Bond, Dietzen, \& Salyers, 1994). In addition, when EBP components or strategies appear spontaneously in routine clinical practice, intensity and potency are often especially weak (Garland et al., 2010).

II. (Ir)relevance of research to practice. A second challenge for DIP and DIS is thatnotwithstanding the studies noted in the previous paragraph—most of our intervention research to date tells us very little about how EBPs will fare in clinical practice. This has limited our ability as a field, and as mentors to the next generation, to understand, convey, and address 
factors related to DI. To illustrate the problem, Table 1 shows a summary based on 461 youth psychotherapy RCTs from the 1060s through 2009. The table shows that that the great majority of treatment trials used recruited (not clinically referred) samples, therapists who were research employees (not practicing clinicians), and university lab or other non-practice settings. Although representativeness has increased somewhat in recent decades, only $2.1 \%$ of all the studies, and only $4.5 \%$ of all studies in the most recent decade, focused on clinically referred clients, treated by practitioners, in clinical practice settings (see also Weisz, Jensen-Doss, \& Hawley, 2005). A similar problem is evident in the research base on treatment integrity (i.e., the extent to which interventions are delivered as intended), which is often noted as a factor explaining the benefit drop-off seen when EBPs are taken to scale in practice. Perepletchikova, Treat, and Kazdin (2007) examined 147 articles, evaluating 202 treatments, in 6 particularly prominent journals (from 2000 through 2004), to assess the extent to which studies established procedures for, assessed, and reported on treatment integrity. Treatment integrity was adequately addressed for only $3.5 \%$ of the tested interventions. Knowledge about the steps taken to ensure treatment integrity and the level of integrity achieved is essential to understanding why treatments fare as they do, particularly when the treatments are taken out of their efficacy trial comfort zone and tested in practice contexts. In particular, investigators need to track integrity to determine whether attenuation of effects in real-world implementation reflects a problem with the intervention itself, or rather with its delivery.

III. Timeline mismatch. A third challenge is the fact that DIS and DIP operate on very different timelines. In DIS, knowledge advances incrementally and at a deliberate pace, with each step requiring time to identify the critical question, develop and refine the study plan, obtain funding, complete the study, identify and interpret findings, and communicate them through 
publication. The published findings may stimulate other studies, then others, in a continuing cascade, but years, even decades may elapse before satisfying answers emerge. By contrast, the timetable for DIP is often tight. Opportunities to disseminate and implement depend on a convergence of policy and funding that may arise quickly and may not last. So, the need to move quickly is legitimate, but this contrasts sharply with the deliberate pace of DIS. The risk is that DIP in real time may proceed without the benefit of DIS, skipping the science needed to identify what it takes to effectively introduce, apply, and sustain EBPs in practice settings.

IV. Goal tensions and implementation limbo. Skipping past DIS may lead to implementation of practices that are not sufficiently tested, using procedures that may not work well, and thus may not actually improve outcomes for targeted populations. The risk may be heightened by the tension between core objectives of DIS (increased understanding of what works) and DIP (action that gets interventions into practice and service settings). Pressures faced by those charged with bringing the interventions into their settings can lead to implementation limbo - the “How low can you go?” approach to DI complexity, time, and cost. DIP often generates bargaining by purchasers—government and agency leaders whose institutional responsibility is to produce as much as possible from a limited pool of funds, and who are working hard to do a good job. In the absence of good evidence on what is required for truly effective DI, a completely understandable impulse is to manage costs. If the developer uses a four-day training but has no evidence that four days is required, why not make it two days? If the developer thinks individual clinician supervision is needed for fidelity, but has no relevant evidence, why not do group supervision? If the developer thinks her experts need to continue doing training and supervision to maintain fidelity and benefit, but has no relevant evidence, why not use a train-the-trainer-and-supervisor model, and save costs by using staff in the local 
setting? The risk is that timetable tensions and implementation limbo will lead to DIP uniformed by DIS, and thus to loss of benefit. As McHugh and Barlow (2010) have noted, “...methods to transport treatments to service delivery settings have developed independently without strong evidence for, or even a consensus on, best practices for accomplishing this task...” (p. 73).

\section{DIP and DIS: Current Practice and Research}

Within the context of these four challenges, we will characterize some of the broad themes of DIP and DIS, focusing on the settings where DI takes place, the methods used, and what is known about the impact of the most common approaches. We will also consider future directions, including strategies that warrant attention, research questions that need to be addressed to enhance success, and the relevance of both to training the next generation.

\section{DIP: Some Current Directions in Dissemination and Implementation Practice}

In this section, we provide examples of DI approaches currently in use, some emerging directions including those related to professional training, and some illustrations of the challenges noted previously.

Research publications. Publishing research findings in peer-reviewed journals is a venerable method for disseminating knowledge about interventions and their effects. Although this may be an effective way to reach researchers, its impact on practitioners is questionable (Stewart \& Chambless, 2007). A recent study suggested that many doctoral-level private practitioners believe treatment outcome research does not generalize well to their populations and settings, and that many do not view published research as pertinent to their practice (Stewart, Stirman, \& Chambless, 2012). This is consistent with survey research indicating that practitioners may rely more on clinical judgment and personal experience than on treatment outcome research (Stewart \& Chambless, 2007). Indeed, even clinicians actively engaged in research perceive empirical 
findings to be of less value in making clinical decisions than other sources of information, including their own clinical experiences and the supervision and consultation they receive (Safran, Abreu, Ogilvie, \& DeMaria, 2011). Thus, although research reports in journals document the empirical foundation of our field, their immediate impact on practice may be modest (Kerner \& Hall, 2009).

Because the evidence base for psychosocial interventions is vast, expert summaries might provide practitioners with access to information that could more readily inform clinical decisions (Straus, Richardson, Glasziou \& Haynes, 2005). Providers who randomly received a summary of research evidence were more likely to recommend evidence-based treatments for hypothetical case vignettes (Raine et al., 2004; Stewart \& Chambless, 2007), but it is not clear how best to make such summary information readily accessible. One approach, to which we now turn, has been to synthesize research findings and disseminate them through electronic media.

Web-based resources. Within the last few decades, the Internet has emerged as a dominant method of disseminating information world-wide. Leveraging the speed and ease of publishing and updating on websites, several organizations have established web-based resources to disseminate information about treatments, evidence on their effects, and implementation methods, to students, trainees, researchers, practitioners, policy-makers, public agencies, and the general public. Table 2 notes some of these organizations, their websites, and key website features.

The American Psychological Association’s (APA) Division 12 (Society of Clinical Psychology) and Division 53 (Society of Clinical Child and Adolescent Psychology), together with the Association for Behavioral and Cognitive Therapies (ABCT) maintain two such websites. Psychotherapies for adults and youths have been reviewed and categorized according 
to level of research support using criteria defined by Chambless and colleagues (1998). At the time this article was written, the two websites listed 74 adult and 46 youth psychotherapies, encompassing a wide range of psychological disorders and problems, as Well-Established or Probably Efficacious. These categories reflect methodological rigor and treatment efficacy, but not treatment effectiveness or readiness for DI.

Other websites maintained by organizations outside the APA and ABCT also list psychosocial interventions that have been evaluated according to each organization's methods and criteria or provide information relevant to DIP and DIS. Two of these, the National Registry of Evidence-Based Programs and Practices (NREPP) and Blueprints for Healthy Youth Development, include information on both methodological rigor and DI readiness (e.g., availability of treatment manuals and implementation protocols, costs). Others such as the What Works Clearinghouse (WWC), and the sibling organizations Cochrane Collaboration and Campbell Collaboration, publish on their websites systematic reviews of interventions, with standardized and well-documented search procedures, inclusion/exclusion criteria, outcome effect sizes, and sometimes meta-analyses to generate mean effect size across intervention studies and identify moderators of treatment benefit. All three websites offer plain-language summaries of the systematic reviews to increase their accessibility, but they do not systematically evaluate DI readiness or provide information to facilitate implementation. In contrast, the National Implementation Research Network (NIRN) and the University of California, San Francisco’s Community Engagement Program maintain websites with information on DI concepts and recommendations for engaging in DIP and DIS.

Continuing education programs. Another widely-used method of spreading knowledge about EBPs comes in the form of continuing education (CE) programs, a primary source of 
training for many practitioners (Addis, 2002). As reported in the 2006 State and Provincial Mandatory Continuing Professional Education Requirements Survey (APA, 2006), CE is required by 43 of 50 states, and the District of Columbia (86\%) for maintaining professional licensure in psychology. States require 10 to 40 CE hours per year, often with the goal of keeping providers updated on new developments in the profession (VandeCreek, Knapp, \& Brace, 1990); in principle, this makes CE programs a good fit to the mission of boosting awareness and use of EBPs. In practice, however, CE program content is often unrelated to the evidence base on effective treatment, and in fact, private practitioners in one study reported that identifying a workshop as covering EBP content might lessen its appeal (Stewart et al., 2012). The primary reasons given for choosing a particular CE program tend to involve cost, convenience, topic, and location (Sharkin \& Plageman, 2003).

Even those CE programs that do focus on EBPs and attract practitioners may not have major impact on practice. Reviews (Beidas \& Kendall,2010; Herschell, Kolko, Baumann, \& Davis, 2010) indicate that training workshops alone typically fail to change clinician behavior or increase proficiency. A study that randomly assigned 115 community therapists to a purely didactic presentation on CBT for anxiety, a more active learning model that included modeling and role-plays of treatment strategies, and computerized training also found limited gains in therapist adherence and skill as assessed by a performance-based role play (Beidas, Edmunds, Marcus, \& Kendall, 2012). On the other hand, training workshops do appear to increase declarative knowledge about EBPs (Beidas \& Kendall, 2010; Beidas et al., 2012). Perhaps CE is better-suited to dissemination than implementation goals.

Government and foundation initiatives. DIP is also carried out through government- and foundation-sponsored initiatives designed to spread tested interventions into routine clinical 
practice (see e.g., McHugh \& Barlow, 2010). One of the largest US initiatives has been spearheaded by the Veterans Health Administration (VHA; Ruzek, Karlin, \& Zeiss, 2012). Thousands of VHA therapists have been trained in EBPs, particularly for trauma (Karlin et al., 2010) and depression (Karlin et al., 2012). At the state level, New York is one of several examples of particularly ambitious DI programs (Hoagwood, Olin, \& Cleek, 2013). In the United Kingdom (UK), a nationwide government-funded DI initiative, Improving Access to Psychological Therapies (IAPT; Clark et al., 2009), is underway to disseminate and implement EBPs for adult anxiety and depression throughout the National Health Service (NHS). Over 1 million patients have received treatment to date, and there are plans to expand the DI initiative to serve youths, older adults, and individuals with severe mental illness, personality disorders, and other conditions (Department of Health, 2012). Private foundations-Annie E. Casey Foundation, MacArthur Foundation, and Robert Wood Johnson Foundation, among others, have supported initiatives to improve mental health by increasing service capacity and effectiveness (see Chambers et al., 2005; Hennessy \& Chambers, 2009).

Some of the initiatives include an effort to build DIS in the context of DIP. For example, in 2002, NIMH introduced a standing program announcement (PA) calling for research applications that would "build knowledge on methods, structures, and processes to disseminate and implement mental health information and treatments into practice settings” (Hennessy \& Chambers, 2009). A second PA focused on communities that had been funded through the Center for Mental Health Services (CMHS), and encouraged research on the implementation of EBPs for children within these grant communities (Chambers, Ringeisen, \& Hickman, 2005). CMHS also funds a \$30 million initiative, the National Child Traumatic Stress Network (NCTSN), with a national coordinating center and 53 individual centers that collaboratively 
develop, implement, evaluate, and disseminate interventions for trauma across all 50 states

(Chambers et al., 2005). In the UK, the National Academy for Parenting Practitioners (NAPP;

Scott, 2010), was created to study, disseminate, and implement parent-mediated EBPs for youth conduct problems.

\section{DIS: Some Current Directions in Dissemination and Implementation Science}

There is a broad consensus that efforts to put EBPs into use in practice settings will need to be guided by empirical evidence on what works — a body of evidence currently best described as under-construction. A systematic review of 495 studies of diffusion of service innovations by Greenhalgh and colleagues (2004) concluded that a lack of knowledge about mechanisms that promote successful service implementation is the "most serious gap in the literature” (p.620). As noted earlier, numerous DIP efforts are underway, but there is wide variation in the extent to which these efforts are guided by theoretical models and empirical findings, or even structured to add to the knowledge base of DIS. Here we offer a sampler of DIS themes and approaches in the research to date.

Effectiveness studies. In effectiveness trials, EBPs previously tested under a high level of experimenter control are tested under conditions more like naturally-occurring clinical care. Effectiveness conditions may include focusing on clients referred through usual community pathways, treatment provided by clinical practitioners not employed by the researcher, and service settings, such as community clinics. An additional effectiveness trial feature for which we have argued (Weisz \& Gray, 2008; Weisz et al., 2006) is random assignment of clients to the EBP being tested vs. representative usual care; this addresses the critical question of whether the EBP actually improves outcomes relative to the intervention that is normally provided in the absence of the EBP. There is a growing body of effectiveness trial evidence with an array of 
treated problems and disorders and across a broad client age range (e.g., Addis et al., 2004;

Weisz et al., 2009). In general, those effectiveness trials in which EBPs are tested against usual care have shown the effect size drop-off described in our earlier section on the implementation cliff. The primary focus of most effectiveness trials is on the impact of previously tested interventions under new and more clinically representative conditions, not on tests of differing strategies for DI; but the experience of leaving the safety of the efficacy context and trying an intervention under less controllable conditions can be a rich source of hypotheses for DIS. A recent analysis, for example, derived from effectiveness research, generated a list of characteristics of "the mental health ecosystem" that can impinge on the use of EBPs in practice contexts, and some testable ideas about how to address them during implementation (Weisz, Ugueto, Cheron, \& Herren, 2013). So, although effectiveness trials may not always contribute directly to DIS, they may have genuine heuristic value.

Systematic reviews of DI studies. There is also heuristic potential in systematic reviews (and potentially meta-analyses) of multiple related DI projects, and such reviews can help characterize the state of the field and identify gaps. As an example, Manuel et al. (2011) conducted a systematic review of 21 studies of the implementation of EBPs for substance use disorders (SUDs). The majority of the studies focused implementation efforts at the individual level (e.g., training counselors) rather than the organizational level (e.g., beginning with a needs assessment, focusing on organization stakeholders, then counselors). Also, very few of the SUD studies used a guiding conceptual model to inform implementation strategies, and thus most did not identify or measure elements that might predict, moderate, or mediate successful EBP implementation. Similarly, a review of studies addressing mental health problems of children in child welfare and mental health settings found that only 9 of the 329 studies identified tested 
specific strategies for implementing an EBP (Landsverk, Brown, Reutz, Palinkas, \& Horwitz, 2011). The findings of both reviews point to DIP that is not only not informed by DIS but also not designed in ways that will help build DIS; but the findings also underscore what is needed, including a theoretical perspective on DI that generates testable hypotheses, which in turn inform the development of study questions and the measurement models needed for an incremental science. Fortunately, some lines of research in our field are already well on the way to taking these steps.

Testing alternate models of DI. One example of such research is the work on Multidimensional Treatment Foster Care (MTFC). Developed as an alternative to group and residential care for delinquent youth with severe emotional and behavioral problems, MTFC has demonstrated benefit in randomized trials with boys referred from juvenile justice (Chamberlain \& Reid, 1998; Eddy \& Chamberlain, 2000), children and adolescents leaving a state hospital setting (Chamberlain \& Reid, 1991), and children in state-supported foster care (Chamberlain, Moreland, \& Reid, 1992). After effectiveness trials that established MTFC as a credible, transportable intervention, researchers are now focusing on DI, to clarify the processes that can facilitate effective implementation and sustainability of MTFC. As described by Chamberlain and Colleagues (2012), three strategies have been used to scale-up MTFC for a large-system use (in counties, states, or nations). In the "rolling cohort” strategy, MTFC is established in a few sites, which in turn help implement MTFC in other sites, with cumulative "lessons learned” integrated into the model in successive sites (Chamberlain et al., 2012). In the "cascading dissemination” strategy, the treatment developers' direct involvement in training and supervision shrinks quickly. The developers train and supervise the initial cohort of interventionists, but once this cohort meets performance criteria for intervention delivery, it trains and supervises the next 
cohort. Finally, the “community development team” (CDT) model encourages peer-to-peer knowledge exchange among systems, organizations, practitioners, and consumers, who are all invited to become members; CDT consultants connect potential adopters with developers and provide encouragement and technical assistance to support adopting and implementing the practice. To support their efforts in using these strategies, MTFC developers created and tested a web-based system for monitoring implementation fidelity, which can then be examined as a possible mediator of treatment outcome (Feil, Sprengelmeyer, Davis \& Chamberlain, 2012). The work of the MTFC team illustrates the promising DIS approach of comparing different implementation strategies within a randomized design (e.g., Saldana \& Chamberlain, 2012). Additional examples can be found in research comparing competing methods for training therapists in Dialectical Behavior Therapy (DBT; Dimeff, Woodcock, Harned, \& Beadnell, 2011), research testing teleconferencing supervision in Motivational Interviewing for Substance Abuse (Smith et al., 2012), and multiple studies of DI methods for Prolonged Exposure Therapy for PTSD (McLean \& Foa, 2011).

Testing specific elements of DI. A useful complement to studies that test entire DI models are studies that test specific DI elements. For example, findings indicating that training workshops alone are not very effective in changing provider behavior (Herschell et al., 2010) have prompted tests of whether other DI components make a difference. Although training workshops alone may not have much impact, training combined with ongoing expert consultation, coaching, or supervision may change provider behavior and other outcomes of interest (Beidas et al., 2012; Herschell et al., 2010). Two RCTs that compared EBP trainings with and without supervision found that providers who also received supervision were more proficient in the newly acquired skills (Miller, Yahne, Moyers, Martinez \& Pirritano, 2004; 
Sholomskas et al., 2005). This suggests, in turn, the need to identify specific supervision practices that increase EBP competency and client outcomes. Green and Seifert (2005) argue that successful implementation of skills requires that newly learned declarative knowledge be translated into well-rehearsed, automatized procedures. This may call for supervision procedures emphasizing role-play and feedback (Bearman et al., in press). Research on this topic is rare, but one study found that "supervision as usual” for clinicians treating disruptive youths used little role play, or even thorough discussion of practices (Accurso, Taylor, \& Garland, 2011). In the future, DIS could profit greatly from studies that manipulate supervision variables and examine changes in clinician behavior, competency, and client outcomes.

Testing sustainability models. Those involved in DIP are concerned not only about whether EBPs can be brought to scale and made effective, but whether-once that is accomplished-the effective practices can be sustained over time. A common pattern is that funds identified for an initial EBP implementation in a service program, region, or state fade out after that initial effort. Are there ways to structure the initial implementation that will give the practices and their benefit holding power after the external funding is gone? Some research suggests that many EBPs are not well-sustained after initial implementation; even when service programs try to sustain them, provider fidelity tends to fade (Stirman et al., 2012). As one possible remedy, some researchers have explored the impact of various 'train the trainer" models in which indigenous agency staff are taught to provide the EBP training and/or supervision after the outside experts are gone. In their cascading implementation research described previously, Chamberlain and colleagues found that "second generation” interventionists (trained and supervised by “first generation” interventionists who had been trained by the treatment developers) achieved fidelity and client outcomes similar to those obtained by the first 
generation interventionists themselves (Buchanan, Chamberlain, Price \& Sprengelmeyer, 2013;

Chamberlain, Price, Reid, \& Landsverk, 2008). Other treatment developers have had less positive experiences with train-the-trainer and second generation models, and have thus retained more prolonged involvement of the intervention developer team (see e.g., Curtis, Ronan, \& Borduin, 2004; Henggeler \& Schaeffer, 2010). Clearly, an important agenda item for DIS will be distinguishing the conditions under which responsibility for training and skill-building in EBPs can and cannot be safely passed on to individuals outside the developer's team.

Examining and manipulating organization-level variables. Organizational factors may influence both the effectiveness and DI of EBPs. Glisson and colleagues (e.g., Glisson, Landsverk et al., 2008) have developed a model of organizational social context (OSC) comprising three factors-climate (i.e., employees’ perceptions of how the work environment affects their well-being and job performance), culture (i.e., employees’ expectations of how work is done), and attitudes (i.e., employees’ job satisfaction and commitment to the organization). This model was supported by data from 1,154 therapists working at a nationally representative sample of 100 US clinics. Importantly, differences in OSC have been linked to DI-related outcomes. Clinics with the best climates had therapist turnover rates that were half that of clinics with the worst climates, and clinics with the best cultures sustained new treatments and services twice as long as clinics with the worst cultures (Glisson, Schoenwald, et al., 2008). Clinics with more engaged and less stressful climates or more proficient cultures had therapists with the most positive attitudes towards using EBPs (Aarons et al., 2012). Furthermore, Glisson and colleagues (2010, 2012) have demonstrated in two RCTs that an organizational intervention can improve (a) the OSC of community mental health service organizations, and (b) the effectiveness and efficiency of MST for delinquent youths. The 2010 study, crossing treatment condition (MST vs. 
usual care) with organizational condition (intervention vs. not) illustrates a particularly innovative way to test organizational enhancement as a strategy for optimizing DI.

Research on the nature of usual practice. The essence of implementation is changing current practice. Hoagwood and Kolko (2009) argue that efforts to change practices that are not fully understood may be “difficult and perhaps foolhardy,” p. 35). This sentiment has prompted a movement to gather “practice-based evidence” (Margison et al., 2000)—data that can help us characterize and document current practices. Certainly a failure to understand typical practices can make it difficult to understand what needs to change, or to find areas of commonality with EBPs. An additional reason to document current practices may be that some of them do not need to be replaced. There is evidence that some usual care treatments may match or exceed the effects of some EBPs (e.g., Wampold et al., 2011; Weisz et al, 2006). Figure 1 illustrates this point: it shows mean effect sizes in a recent collection of 52 RCTs comparing youth EBPs to usual care. The figure shows that in 13 of the studies, there was either a negligible difference in outcome or a superior outcome for usual care. Unfortunately, most studies that have included a usual care condition have not investigated what usual care consisted of; thus, when usual care has shown null effects we do not know what needs to be improved, and when usual care has outperformed EBPs we do not know what the procedures were that fared so well and might bear further testing. Fortunately, investigators have recently developed coding systems for documenting the contents of usual care (see e.g., McLeod \& Weisz, 2005, 2010), and some have begun to apply coding to usual care session recordings (Bearman et al., 2011; Garland et al., 2010). The work is likely to be complex, in part because the nature of usual care will differ across client groups, providers, and intervention settings. However, in planning implementation studies and interpreting their findings, a capacity to describe the usual care context within which 
the studies took place may prove invaluable. Recent research has also used observational methods to characterize typical supervision processes in community clinics (Accurso et al., 2011). If (as seems likely) clinical supervision is critical to success in DIP, sound methods will be needed for characterizing it in a variety of contexts.

Research on subjective experience of “end users.” Because DIP is all about engagement of key stakeholders, including providers, clinic staff, and clients, it is important to understand their subjective response to DI efforts. As we noted, research findings may do little to sway a provider’s opinion. Put another way, “a bridge between research and practice is not likely to come from a finding that refutes a specific concern about an EBP ('See, we told you that comorbid patients can be treated effectively.')...” (Kazdin, 2008, p.150). To really understand the subjective experience of key stakeholders, and what factors make change acceptable when change is needed, we need research that focuses directly on that subjective experience. Some of this work may require qualitative methods, which can generate testable hypotheses about facilitators of DI in service settings (Palinkas et al., 2011). Some excellent examples of this work include a qualitative interview study of doctoral-level private practitioners (Stewart et al., 2012) and an ethnographic study of public agency therapists participating in a community effectiveness trial (Palinkas et al., 2008). As an example of what can be learned, Palinkas et al. (2008) found that one of the most significant factors in clinician decisions about whether to continue using a new treatment after training was the lag time between initial training in the protocol and the first case assignment that was appropriate for the protocol. This suggests the hypothesis that having appropriate cases ready for assignment soon after training could enhance clinicians' willingness, and thus the success of implementation. More generally, the qualitative studies have suggested that therapists prefer treatment approaches that can be tailored to meet the 
needs of their clients and integrated flexibly into their existing practices. Broadening the focus to include clients and other stakeholders will further enrich our picture of end users as key players in the DI process.

\section{Training Programs as Avenues for Advancing DIP and DIS}

Training programs for graduate students, interns, and postdoctoral fellows in clinical psychological science, and in related mental health professions, may offer multiple avenues for advancing DIP and DIS. First, clinical training, practica and internships may include hands-on DIP with trainees. Second, training programs can be sites for DIS by offering opportunities to study what works and doesn't in DIP. Third, training programs may teach DI concepts and issues to trainees in ways that support careers in DIP or DIS. In this section, we focus on these capacities of training programs, and some of the relevant evidence.

Training programs as DI sites. Training programs, by their very nature, are active DIP sites, but which treatments do they disseminate and implement? A survey of US psychotherapy training programs (Weissman et al., 2006) revealed that 67\% of Psy.D. programs and 44\% of clinical psychology Ph.D. programs did not require coursework and clinical supervision in even one EBP (compared to only $4 \%$ of psychiatry residency programs and $62 \%$ of social work programs). A survey of APA-accredited Ph.D. and Psy.D. programs and internships in North America (Woody, Weisz, \& McLean, 2005) found that most doctoral and internship programs provided supervised training in no more than 4 to 5 of all the available ESTs, with little agreement across training programs about which treatments should be taught. Finally, in a survey of graduate students from clinical, counseling, and school psychology programs in North America (Karekla, Lundgren, \& Forsyth, 2004), 65\% of clinical psychology graduate students reported not having read any publications by the APA Division 12 Task Force on the Promotion 
and Dissemination of Psychological Procedures on EBPs, and 25\% reported not using EBPs in their clinical training, although half the graduate students reported that they intended to use EBTs in their future clinical work. Surveys of training directors note such perceived obstacles to EBP training as lack of trainee interest, scarcity of qualified instructors and supervisors, doubts that EBPs are relevant to or effective in clinical practice, and philosophical disagreements including the perceptions that EBPs represent theoretical bias or undermine psychologists' autonomy (see Weissman et al., 2006; Woody et al., 2005). An additional obstacle is that so much clinical training takes place in external practicum sites over which doctoral programs have little control (see Lewis, Hatcher, \& Pate, 2005; Woody et al., 2005). The Weissman et al. (2006) findings suggest that we might learn from psychiatry training programs, which were more likely to report obstacles than psychology programs, but which also reported more ultimate success, reportedly because of accreditation changes that required EBP training.

Training programs as career-building sites for DI practitioners and scientists. Training programs can also prepare students to be DI practitioners and scientists, and some already do this. A special issue of The Behavior Therapist (Hawkins, 2002) featured several programs’ efforts to integrate science, practice, and community service. McMahon, Ribordy, and Washburn (2002), for example, described a collaboration between a training clinic and schools in the community to train graduate students in conducting school-based intervention and research. In addition, Chu et al. (2012) proposed a model to prepare psychologists for leadership roles in community mental health settings through coursework, training collaborations with community clinics, and group case-method learning (e.g., write proposals to disseminate an EBP within a community clinic). Finally our own labs, like a number of others, conduct treatment studies in which community therapists are trained in EBPs, and examine both treatment outcomes and 
implementation outcomes. Postdoctoral fellows in these studies learn a range of the skills needed for careers in DIP and DIS.

\section{Agenda for the Future: Directions for Practice, Research, and Training}

DIP and DIS are far more extensive and diverse than our brief sampler can convey. But the examples illustrate the rich array of approaches and topics assembled beneath these two massive umbrellas. Having noted some core challenges and illustrated some of the content of DIP and DIS, we turn now to directions for the future, in practice, research, and training (summarized in Table 3).

\section{Making Intervention Research Look More Like Practice}

We noted at the beginning of this paper that DIP tends to move on a rapid timeline, often far ahead of the knowledge base provided by DIS, and that our intervention research often shows low relevance to actual clinical practice. These two problems are not unrelated. The problem of low relevance is in fact one reason the DIS knowledge base struggles so to keep up with the pace of DIP. We have argued (e.g., Weisz, 2004; Weisz \& Gray, 2008) for a deployment-focused model within which interventions are developed and tested, as soon as feasible, with the kinds of clients and therapists, and in the kinds of settings, for which the interventions are ultimately intended. If this model were followed, the research strategy known as efficacy testing would be only a brief initial phase in intervention development, used to establish the potential for benefit, and effectiveness testing under clinically representative conditions would be the dominant research approach. If we want to prepare our students for a professional world in which DI is a central theme, and we want their work to be guided by a vibrant and relevant science, it may be wise to consider the potential advantages of the deployment-focused model for both research and clinical training. 


\section{Solving the “Too Many EBPs" Problem}

A quick trip to any of the web-based clearinghouses and resources cited above will make it clear that many intervention programs are now classified as “evidence-based,” and very likely far more than could be effectively implemented at a population level. The NREPP website (see above), for example, lists more than 280 “evidence-based” interventions. Becoming skilled at even one intervention can be a multi-year process, even for a graduate student who may learn only one program in depth, and certainly for a busy practitioner with a large caseload and very limited time for additional learning. Stated briefly, intervention science has generated a list of EBPs that DIP may not be able to accommodate, and that DIS would have great difficulty studying adequately. The long list is thinned somewhat by the thoughtful addition to NREPP of information on "implementation history” and "readiness for dissemination;" but it is possible that DIS can generate still more rigorous standards for which interventions warrant the considerable time, effort, and expense of DIP. As one example, a case can be made that the only interventions that should supplant usual care are those that have been shown to produce reliably better outcomes than usual care in fair, randomized trial comparisons. If this standard were applied, the findings of recent meta-analyses (Wampold et al., 2011; Weisz et al., 2006) suggest that a very short list of interventions would survive_-a list that might actually be manageable as a target for both DIP an DIS. A challenge for our next generation, currently in training, will be to develop and apply thoughtful criteria in determining which of the "evidence-based” interventions truly warrant DIP and thus need to be part of the subject matter of DIS. For this work we need to engage the best and the brightest among those we are now training.

\section{Addressing the Mismatch between Interventions and Users}


The "too many EBPs" problem may also be alleviated somewhat by efforts to address another problem for DIP: many of the interventions classified as EBPs are designed in ways that may not be an ideal fit to their users - both therapists and clients. For example, most EBPs are built for a single disorder or a small homogeneous cluster. In contrast, most clinicians carry very diverse caseloads, such that becoming expert in one EBP for one disorder is relevant to only a tiny portion of their clientele. The single-disorder nature of many EBPs also conflicts to a degree with the fact that most referred clients have comorbid disorders that need attention, and that the most pressing problems that need attention can shift during treatment, rendering the single focus with which treatment began no longer so relevant. These mismatches can lead to frustration among both therapists and clients, undermining the sustainability of new practices and discouraging future participation in DIP. One approach to addressing the problems of diverse caseload, client comorbidity, and flux in treatment is to build integrative treatments that combine intervention components from EBPs originally developed for different problems that are often comorbid. Barlow and colleagues (2011) have developed a transdiagnostic unified protocol for the treatment of emotional disorders across the affective-anxiety spectrum in adults; an initial RCT with adults who had a primary anxiety disorder (Farchione et al., 2012) showed superior outcomes relative to waitlist controls, with treatment gains maintained during 6-month followup. Chorpita and Weisz (2009) have developed a modular protocol for the treatment of child and adolescent disorders and problems involving anxiety, depression, and disruptive conduct; in a recent effectiveness trial, this protocol produced significantly better clinical outcomes than (a) usual outpatient care and (b) conventional single-disorder protocols (Weisz et al, 2012). Such integrative treatments may also increase efficiency, reducing the need for multiple trainings and juggling of multiple single-disorder protocols. Ideally, the skill set of the next generation in our 
field will include creative intervention design, combining the invaluable work of treatment developers to date with new ideas for treatment organization and structure to fit the characteristics and needs of their users, and testing whether the redesigned interventions really work in representative clinical care contexts. In addition, we will need research on mechanisms of change, to inform decisions about which components to retain and discard (Kazdin \& Blase, 2011), and research on patient attributes that moderate treatment effects, to tell us for whom these simplified treatments are effective, and for whom more intensive interventions are required (Shoham \& Insel, 2011).

\section{Building Efficient and Accessible Delivery Methods and Models}

Another way to repackage EBPs is to supplement current delivery methods and models with more efficient and accessible ones. Traditionally a patient sees a highly-trained therapist individually in an office for an hour a week; this model may be inaccessible, unaffordable, unappealing (e.g., due to stigma or lack of engagement), or otherwise logistically unworkable for many. Kazdin \& Blase (2011) have advocated a "portfolio" of treatment delivery methods to increase the public-health impact of psychotherapies (see also Rotheram-Borus, Swedeman, \& Chorpita, 2012). These methods could include self-help books that impart EBP-related knowledge and skills to patients (e.g., Burns, 1980; see Stice, Burton, Bearman, \& Rohde, 2006), interactive computerized versions of EBPs (e.g., Merry et al., 2012), video-guided treatments with reduced therapist involvement (e.g., Webster-Stratton \& Reid, 2010), individual treatment with a therapist through phone or videoconferencing (e.g., Morland et al., 2010), low-intensity therapies conducted by paraprofessionals (e.g., Clark et al., 2009), and therapies embedded within everyday settings such as summer camps (e.g., Pelham et al., 2010). Such approaches may increase the DI of EBPs by reducing the cost, travel time, logistical barriers, and stigma 
associated with traditional treatment delivery methods, and by increasing engagement in certain populations (e.g., children drawn to computers). Some of these alternative delivery methods have demonstrated efficacy, but much more research will be needed to test effectiveness in everyday conditions and to identify the delivery formats that best balance cost-effectiveness and clinical benefit (see Yates, 2011). The call for more efficient and accessible treatment methods fits well within a public health model comprised of multiple tiers (see Atkins \& Frazier, 2011), with lower intensity-broader reach interventions for low-risk, low-symptom groups, and higher intensity interventions for high-risk, high-symptom groups. Examples of this approach include the UK-wide IAPT (e.g., Clark et al., 2009) initiative described earlier, and the Triple P-Positive Parenting Program, an internationally disseminated EBP (Sanders, 2012).

\section{Solving the “How'm I Doing?” Problem}

Implementing a new intervention can be nerve-wracking for a therapist. Knowing how the client is responding, on a regular basis, can facilitate implementation by helping the therapist know when things are going well or not, and decide when to change strategies and when to end treatment. Thus, another skill on the forefront of DIP and DIS, and valuable for the next generation of professionals, is the design and implementation of information systems that provide feedback on client response. Such a system was used in conjunction with implementation of the modular protocol for youth, described earlier; the system provided weekly feedback on youth client progress throughout treatment using two brief, psychometrically sound measures (Chorpita et al., 2010; Weisz et al., 2011), displayed on a “client dashboard” within an internet-based system. Recent research has shown that providing such client progress feedback to clinicians, even in the absence of EBPs, may improve therapy success and reduce deterioration among adults (Lambert et al., 2002; Anker, Duncan, \& Sparks, 2009), and improve therapeutic 
relationships and outcomes among youths (Bickman, Kelley, Breda, deAndrade, \& Riemer, 2011; Stein, Kogan, Hutchison, Magee, \& Sobero, 2010).

\section{Embracing High-Risk/High-Gain Innovation and Learning from Failure}

In April, 2011, the Harvard Business Review published a “failure issue,” packed with examples of the rich lessons learned from business flops. We, and our students, might consider this perspective in the context of the fourth challenge noted at the beginning of this paper: Implementation limbo. DIP carries the seemingly inevitable push to see "How low can you go?" on time, effort, and cost in bringing tested interventions to scale. This push from funders and administrators is often quite fair and reasonable — they are doing their job; indeed, our job may well need to include finding cost-effective ways to disseminate and implement, because these will maximize the number who may benefit. Moreover, high-risk/high-gain ventures testing daring new DI strategies may generate real payoff and potent new approaches. The downside is that playing implementation limbo and taking risks with daring innovations virtually guarantees frequent failures — tests of leaner or more novel ways to deliver treatments that ultimately prove unsuccessful. Each failure may add important information on what doesn't work—an important complement to learning what does work, because both help set boundaries guided by science. And as any meta-analyst would note, reporting failures is critical to a balanced picture of intervention effects; woe be the science in which only positive results are published. This is a nice philosophical perspective, but will it work as a career path and a training model? Will our graduate students and postdocs be respected for the high quality of their research if much of that research involves learning through failure? Will prominent journals publish their null findings if the studies have pristine design? Will our students land positions at competitive universities, and be promoted and tenured, if their treatment trials do not show a string of substantial positive 
effects? These are important questions for our profession as we move into an era dominated by DIP and DIS, with limbo music in the background.

\section{Contemplating New Rules and Professional Tracks}

With the growth of DIP, the potential costs of failed trials may now be both professional and financial. The challenge we face, increasingly with each passing year, is that enormous incentives — financial and other-attach to the business of DI. Professionals trained to be scientists or practitioners are going into business, starting corporations, and marketing the spread of interventions. As the businesses grow, the cost of a null or adverse outcome extend to more and more lives, with ever-greater financial implications, and with concomitant pressure to design projects for success, emphasize the positive aspects of mixed findings, and market by focusing on the good news and shading the bad. Under these circumstances, some see a risk of "creeping pharma,” a situation in which the massive incentives available through successful marketing may swamp the rigor and caution needed for scientifically sound information on intervention effects and limitations. We may need to consider, as a field and as mentors, whether new rules or ethical guidelines are needed to ensure fairness and openness, for the benefit and protection of those who will receive the interventions being purveyed. We may also consider specialty career paths designed to provide balance in a world tilting increasingly toward profit. For example, do we need to create career tracks for "trialists," students who will commit to not pursuing a developer stake or income from sales, but whose only role, supported by a career incentive system, will be to ferret out the truth by designing patently fair tests of interventions? Do we need to create career tracks for “evidence brokers,” students committed to conducting systematic reviews and meta-analyses, in as fair a way as possible, and with no allegiance to any intervention they are 
examining? [If so, we would also need to create funding streams to make more of this synthesizing work possible, since grant funding for such work is rare, at present.]

The Cochrane and Campbell Collaborations represent significant efforts to support evidence broker tracks in healthcare and social intervention research. Both list all funding sources on their websites and require prospective authors to collaborate with a Review/Coordinating Group that provides editorial and methodological support and oversight throughout the conduct of a review. In addition, Cochrane refuses funding from commercial organizations, and reviews must have at least one author who was not involved any of the studies reviewed. A procedural handbook is posted online and regularly updated with input from methodological experts. Cochrane and Campbell are exemplary in several ways, but most systematic reviews and meta-analyses continue to be published in academic journals that cannot provide the same level of scrutiny, quality control, or detailed reporting. Importantly, both Cochrane/Campbell reviews and those in journals are most often focused on treatment efficacy, not on effectiveness, tests of EBPs vs. usual care, or readiness to be taken to scale- the themes most relevant to DIS.

\section{Deciding Who Owns the Cure}

This concern about the business of DIP, and the risks it may pose to our scientific integrity, may need to be balanced against issues of ownership and its risks. Here we refer to the complicated set of issues around "Intellectual Property" as it relates to our field. There is now a confusing mix of policies, laws, and court rulings that often leave it unclear who "owns" the “products” associated with EBPs. Should corporations, universities, and dissemination companies own these products and market them, or should ownership and control be vested in those who have developed the interventions and arguably know their strengths and limitations, 
and the kinds of training and skill-building needed to ensure their effective implementation? Both kinds of ownership may pose significant risks. Our contribution could be a scientific perspective on what will ultimately do the most good for those who need mental health care. Our perspective, and the evidence on which it is based, might be used to guide funding agencies in their decisions about which ownership arrangements will ultimately do the most good for clients. These decisions could have a marked impact on DIP and DIS in generations well beyond our own.

\section{Building Community-Research Partnerships}

A process that may be needed for optimum DIP and DIS, and one that may need to be targeted in our training programs, is building community-research partnerships, in which decision making is shared by professionals and community members. The Community-Based Participatory Research approach (CPR; Israel, Eng, Schulz, \& Parker, 2005) assumes that, just as the professionals may have EBP expertise, community partners have expertise in the target community's current status, goals, resources, and needs, and that each area of expertise is critical for DI (Becker, Stice, Shaw, \& Woda, 2009). Applying this perspective, (a) Becker et al (2009) used CPR to adapt and implement a prevention program for eating disorders within a large sorority, (b) Brookman-Frazee, Stahmer, Lewis, Feder, and Reed (2012) used CPR to identify, implement, and sustain an EBP for infants and toddlers at risk for Austism Spectrum Disorders in a community clinic, and (c) Bradshaw et al. (2012) used a CPR partnership between the Maryland Department of Education, Sheppard Pratt Health System, and Johns Hopkins University, to build a strategy for implementing a program preventing disruptive behavior, in 800 public schools. As DIP and DIS grow in scope and complexity, the need for skills in CPR 
may grow ever more pressing. As noted earlier, some CPRs also serve training functions that can have long-term value for our discipline (Chu et al., 2012).

\section{Courtship and Marriage of DIP and DIS}

Finally, we suggest that the timeline challenge, noted early in the paper, the fact that DIP often leaps into action ahead of DIS, could be addressed in part through a marriage of DIP and DIS. Massive amounts of government and foundation funding are spent each year on demonstration projects which are essentially DIP with no hint of DIS. Under these circumstances, the separation is reinforced, and the gap sustained. Suppose, instead, we were to adopt a policy that every demonstration project must be designed to answer a useful scientific question about DI. This mandate would turn every demonstration project into a learning opportunity by pushing the designers to identify questions that could be answered through strategically selected comparisons. It is worth recalling that the System of Care model of child and family intervention was dominant and unchallenged in the US for many years, with claims of great success based on unevaluated demonstration projects. It was only when an evaluation component was added that Bickman and colleagues (see Bickman, 1996; Bickman et al., 1999) discovered that system of care increased costs but not benefit. Beyond treatment outcome comparisons, a variety of additional question of real value to DIS can be added to studies of intervention implementation, thus magnifying the value of the projects and often with little added cost (see Schoenwald, 2010; Sanders, 2012).

\section{Concluding Comment}

In this paper we have noted risks posed by a close relation between DIP and DIS, but we have also noted many ways the two complement, inform, and enhance one another. A clean separation of practice and science - in projects, personnel, etc.—might offer advantages, 
including protecting the science from the influence of vested interests. However, we cannot avoid the fact that DIP is in fact the subject matter of DIS, and a close connection is needed to ensure that practice will be evaluated and guided by science while science is informed by the questions that arise in practice. In DI, it appears that science and practice are both a cute couple and strange bedfellows; their shared interests and need for one another will likely make them an inseparable couple, but their relationship will need to be watched closely and managed carefully in the days ahead, just like any relationship between two strong partners. 


\section{References}

Aarons, G. A., Glisson, C., Green, P. D., Hoagwood, K., Kelleher, K. J., Landsverk, J. A., \& The Research Network on Youth Mental Health. (2012). The organizational social context of mental services and clinician attitudes toward evidence-based practice: A United States national study. Implementation Science, 7: 56. doi:10.1186/1748-5908-7-56

Accurso, E. C., Taylor, R. M., \& Garland, A. F. (2011). Evidence-based practices addressed in community-based children's mental health clinical supervision. Training and Education in Professional Psychology, 5, 88-96. doi: 10.1037/a0023537

Addis, M. E. (2002). Methods for disseminating research products and increasing evidencebased practice: Promises, obstacles, and future directions. Clinical Psychology: Science and Practice, 9, 367-378. doi: 10.1093/clipsy/9.4.367

Addis, M. E., Hatgis, C., Krasnow, A. D., Jacob, K., Bourne, L., \& Mansfield, A. (2004). Effectiveness of cognitive-behavioral treatment for panic disorder versus treatment as usual in a managed care setting. Journal of Consulting and Clinical Psychology, 72, 625-635. doi: 10.1037/0022-006X.72.4.625

American Psychological Association. (2006). State provincial mandatory continuing education in psychology (MCEP) requirements — 2006 survey results. Retrieved from http://www.apa.org/ed/sponsor/resources/requirements.aspx

Anker, M. G., Duncan, B. L., \& Sparks, J. A. (2009). Using client feedback to improve couple therapy outcomes: A randomized clinical trial in a naturalistic setting. Journal of Consulting and Clinical Psychology, 77, 693-704. doi: 10.1037/a0016062

Atkins, M. S., \& Frazier, S. L. (2011). Expanding the toolkit or changing the paradigm: Are we ready for a public health approach to mental health? Perspectives on Psychological Science, 
6, 483-487.

Barlow, D. H., Farchione, T. J., Fairholme, C. P., Ellard, K. K., Boisseau, C. L., Allen, L. B., \& Ehrenreich-May, J. (2011). The unified protocol for transdiagnostic treatment of emotional disorders: Therapist guide. New York: Oxford University Press.

Bearman, S. K., Herren, J., Dorf-Caine, R., Michl, L., Zoloth, E., Jhe, G., \& Weisz, J.R. (2011, November). Taking Care (With Usual Care): Common elements across evidence-based treatments for youth depression and treatment as usual in school settings in C. K. HigaMcMillan (Chair) and A. Garland (Discussant), Comparing Usual Care and Evidence-Based Care for Children and Adolescents: Advancing Dissemination in the 21st Century. Symposium presented at the annual meeting of the Association of Behavioral and Cognitive Therapies, Toronto, Canada.

Bearman, S. K., Weisz, J. R., Chorpita, B. F., Hoagwood, K., Ward, A., Ugueto, A. M., \& Bernstein, A. (in press). More practice, less preach? The role of supervision processes and therapist characteristics in EBP implementation. Administration and Policy in Mental Health and Mental Health Services Research.

Becker, C. B., Stice, E., Shaw, H., \& Woda, S. (2009). Use of empirically supported interventions for psychopathology: Can the participatory approach move us beyond the research-to-practice gap? Behaviour Research and Therapy, 47, 265-274.

Beidas, R. S., Edmunds, J. M., Marcus, S. C., \& Kendall, P. C. (2012). Training and consultation to promote implementation of an empirically supported treatment: A randomized trial. Psychiatric Services, 63, 660-665.

Beidas, R. S., \& Kendall, P. C. (2010). Training therapists in evidence-based practice: A critical review of studies from a systems-contextual perspective. Clinical Psychology: Science and 
Practice, $17,1-30$.

Bickman, L. (1996). A continuum of care: More is not always better. American Psychologist, 51, $689-701$.

Bickman, L., Kelley, S. D., Breda, C., deAndrade, A., \& Riemer, M. (2011). Effects of routine feedback to clinicians on mental health outcomes of youths: Results of a randomized trial. Psychiatric Services, 62, 1423-1429.

Bickman, L., Noser, K., \& Summerfelt, W. T. (1999). Long-term effects of a system of care on children and adolescents. Journal of Behavioral Health Services Research, 26, 185-202.

Bradshaw, C. P., Pas, E. T., Bloom, J., Barrett, S., Hershfeldt, P., Alexander, A., . . Leaf, P. J. (2012). A state-wide partnership to promote safe and supportive schools: The PBIS Maryland initiative. Administration and Policy in Mental Health and Mental Health Services Research, 39, 225-237. doi: 10.1007/s10488-011-0384-6

Brookman-Frazee, L., Stahmer, A. C., Lewis, K., Feder, J. D., \& Reed, S. (2012). Building a research-community collaborative to improve community care for infants and toddlers at-risk for autism spectrum disorders . Journal of Community Psychology, 40, 715-734. doi:10.1002/jcop.21501

Buchanan, R., Chamberlain, P., Price, J. M., \& Sprengelmeyer, P. (2013). Examining the equivalence of fidelity over two generations of KEEP implementation: A preliminary analysis. Children and Youth Services Review, 35, 188-193.

doi:10.1016/j.childyouth.2012.10.002

Burns, D. (1980). Feeling good. New York: Signet.

Chamberlain, P., Moreland, S., \& Reid, K. (1992). Enhanced services and stipends for foster parents: Effects on retention rates and outcomes for children. Child Welfare: Journal of 
Policy, Practice, and Program, 71, 387-401.

Chamberlain, P., \& Reid, J. B. (1991). Using a specialized foster care community treatment model for children and adolescents leaving the state mental hospital. Journal of Community Psychology, 19, 266-276. doi: 10.1002/1520-6629(199107)19:3<266::AIDJCOP2290190310>3.0.CO;2-5

Chamberlain, E, \& Reid, J. (1998). Comparison of two community alternatives to incarceration for chronic juvenile offenders. Journal of Consulting and Clinical Psychology, 6, 624- 633.

Chamberlain, P., Price, J. Reid, Landsverk, J. (2008). Cascading implementation of a foster and kinship parent intervention. Child Welfare, 87, 27-48.

Chamberlain, P., Roberts, R., Jones, H., Marsenich, L., Sosna, T., Price, J. M. (2012). Three collaborative models for scaling up evidence-based practices. Administration and Policy in Mental Health and Mental Health Services Research, 39, 278-290. doi: 10.1007/s10488011-0349-9

Chambers, D. A., Ringeisen, H., \& Rickman, E. E. (2005). Federal, state, and foundation initiatives around evidence-based practices for child and adolescent mental health. Child and Adolescent Psychiatric Clinics of North America, 14, 307-327.

Chambless, D. L., Baker., M. J., Baucom, D. H., Beutler, L. E., Calhoun, K. S., Crits-Christoph, P., . . W Woody, S. R. (1998). Update on empirically validated therapies II. The Clinical Psychologist, 51, 3-15.

Chorpita, B. F., \& Weisz, J. R. (2009). MATCH-ADTC: Modular approach to therapy for children with anxiety, depression, trauma, or conduct problems. Satellite Beach, FL: PracticeWise. 
Chorpita, B. F., Reise, S., Weisz, J. R., Grubbs, K., Becker, K. D., Krull, J. L., \& the Research Network on Youth Mental Health. (2010). Evaluation of the brief problem checklist: Child and caregiver interviews to measure clinical progress. Journal of Consulting and Clinical Psychology, 78, 526-536.

Chu, J. P., Emmons, L., Wong, J., Goldblum, P., Reiser, R., Barrera, A. Z., \& Byrd-Olmstead, J. (2012). The Public Psychology Doctoral Training Model: Training clinical psychologists in community mental health competencies and leadership. Training and Education in Professional Psychology, 6, 76-83. doi: 10.1037/a0028834

Clark, D. M., Layard, R., Smithies, R., Richard, D. A., Suckling, R., \& Wright, B. (2009). Improving access to psychological therapy: Initial evaluation of two UK demonstration sites. Behaviour Research and Therapy, 47, 910-920. doi:10.1016/j.brat.2009.07.010

Curtis, N. M., Ronan, K. R., \& Borduin, C. M. (2004). Multisystemic treatment: A meta-analysis of outcome studies. Journal of Family Psychology, 18, 411-419. doi: 10.1037/08933200.18.3.411

Department of Health. (2012, November). IAPT three-year report: The first million patients. Retrieved from http://www.iapt.nhs.uk/silo/files/iapt-3-year-report.pdf

Dimeff, L. A., Woodcock, E. A., Harned, M. S., \& Beadnell, B. (2011). Can dialectical behavior therapy be learned in highly structured learning environments? Results from a randomized controlled dissemination trial. Behavior Therapy, 42, 263-275. doi: 10.1016/j.beth.2010.06.004

Eddy, J. M., \& Chamberlain, R (2000). Family management and deviant peer association as mediators of the impact of treatment condition on youth antisocial behavior. Journal of Consulting and Clinical Psychology, 68, 857-863. 
Farchione, T. J., Fairholme, C. P., Ellard, K. K., Boisseau, C. L., Thompson-Hollands, J., Carl J. R., . . . Barlow, D. H. (2012). The unified protocol for the transdiagnostic treatment of emotional disorders: A randomized controlled trial. Behavior Therapy, 3, 666-678.

Feil, E. G., Sprengelmeyer, P. G., Davis, B., \& Chamberlain, P. (2012). Development and testing of a multimedia Internet-based system for fidelity and monitoring of multidimensional treatment foster care. Journal of Medical Internet Research, 14, 239-248.

Foa, E. B., Hembree, E. A., Cahill, S. P. Rauch, S. A. M., Riggs, D. S., Feeny, N.C., \& Yadin, E. (2005). Randomized trial of prolonged exposure for posttraumatic stress disorder with and without cognitive restructuring: Outcome at academic and community clinics. Journal of Consulting and Clinical Psychology, 73, 953-964.

Garland, A. F., Brookman-Frazee, L., Hurlburt, M. S., Accurso, E. C., Zoffness, R. J., HaineSchlagel, R., \& Ganger, B. (2010). Mental health care for children with disruptive behavior problems: a view inside therapists' offices. Psychiatric Services, 61, 1-8.

Glisson, C., Hemmelgarn, A., Green, P., Dukes, D., Atkinson, S., \& Williams, N. J. (2012). Randomized trial of the Availability, Responsiveness, and Continuity (ARC) organizational intervention with community-based mental health programs and clinicians serving youth. Journal of the American Academy of Child and Adolescent Psychiatry, 51, 780-787.

Glisson, C., Landsverk, J., Schoenwald, S., Kelleher, K., Hoagwood, K. E., Mayberg, S., \& Green, P. (2008). Assessing the organizational social context (OSC) of mental health services: Implications for research and practice. Administration and Policy in Mental Health and Mental Health Services Research, 35, 98-113. doi: 10.1007/s10488-007-0148-5

Glisson, C., Schoenwald, S. K., Hemmelgarn, A., Green, P., Dukes, D., Armstrong, K. S., \& Chapman, J. E. (2010). Randomized trial of MST and ARC in a two-level evidence-based 
treatment implementation strategy. Journal of Consulting and Clinical Psychology, 78, 537550. doi: 10.1037/a0019160

Glisson, C., Schoenwald, S. K., Kelleher, K., Landsverk, J., Hoagwood, K. E., Mayberg, S., .. . The Research Network on Youth Mental Health. (2008). Therapist turnover and new program sustainability in mental health clinics as a function of organizational culture, climate, and service structure. Administration and Policy in Mental Health and Mental Health Services Research, 35, 124-133. doi 10.1007/s10488-007-0152-9

Greenhalgh, T., Macfarlane, F., Bate, P. Kyriakidou, O. (2004). Diffusion of innovations in service organizations: systematic review and recommendations. The Milbank Quarterly, 82, $581-629$.

Green, L. A., \& Seifert, C. M. (2005). Translation of research into practice: Why we can’t “just do it”. Journal of the American Board of Family Medicine, 18, 541-545. doi: 10.3122/jabfm.18.6.541

Hawkins, R. C. (2002). Behavior therapy and the training clinic: Can science be integrated with practice and community service? The Behavior Therapist, 25, 97-98.

Henggeler, S. W., \& Schaeffer, C. (2010). Treating serious antisocial behavior using Multisystemic Therapy. In J. R. Weisz \& A. E. Kazdin (Eds.), Evidence-based psychotherapies for children and adolescents (2nd ed., pp. 259-276). New York, NY: Guilford Press.

Hennessy, K., \& Chambers, D. A. (2009). Delivery of excellent mental health care and acceleration of research: Federal activities since the President’s Commission Report. Psychiatric Services, 60, 433-438. doi: 10.1176/appi.ps.60.4.433

Herschell, A. D., Kolko, D. J., Baumann, B. L., \& Davis, A. C. (2010). The role of therapist 
training in the implementation of psychosocial treatments: A review and critique with recommendations. Clinical Psychology Review, 30, 448-466.

Hoagwood, K., \& Kolko, D. J. (2009). Introduction to the special section on practice contexts: A glimpse into the nether world of public mental health services for children and families. Administration and Policy in Mental Health and Mental Health Services, 36, 35-36.

Hoagwood, K., Olin, S., \& Cleek, A. (2013). Beyond context to the skyline: Thinking in 3D. Administration and Policy in Mental Health and Mental Health Services Research, 40, 2328.

Israel, B. A., Eng, E., Schulz, A. J., Parker, E. A. (2005). Methods in community-based participatory research for health. San Francisco: Jossey-Bass.

Karekla, M., Lundgren, J. D., \& Forsyth, J. P. (2004). A survey of graduate training in empirically supported and manualized treatments: A preliminary report. Cognitive and Behavioral Practice, 11, 230-242.

Karlin, B. E., Brown, G. K., Trockel, M., Cunning, D., Zeiss, A. M., \& Taylor, C. B. (2012). National dissemination of cognitive behavioral therapy for depression in the department of veterans affairs health care system: Therapist and patient-level outcomes. Journal of Consulting and Clinical Psychology, 80, 707-718. doi: 10.1037/a0029328

Karlin, B. E., Ruzek, J. I., Chard, K. M., Eftekhari, A., Monson, C. M., Hembree, E. A., ... Foa, E. B. (2010). Dissemination of evidence-based psychological treatments for posttraumatic stress disorder in the Veterans Health Administration. Journal of Traumatic Stress, 23, 663673. doi:10.1002/jts.20588

Kazdin, A. E. (2008). Evidence-based treatment and practice: New opportunities to bridge clinical research and practice, enhance the knowledge base, and improve patient care. 
American Psychologist, 63, 146-159. doi: 10.1037/0003-066X.63.3.146

Kazdin, A. E. \& Blase, S. L. (2011). Rebooting psychotherapy research and practice to reduce the burden of mental illness. Perspectives on Psychological Science, 6, 21-37. doi: 10.1177/1745691610393527

Kerner, J. F. \& Hall, K. L. (2009). Research dissemination and diffusion: Translation within science and society. Research on Social Work Practice, 19, 519-530. doi: 10.1177/1049731509335585

Lambert, M. J., Whipple, J. L., Vermeersch, D. A., Smart, D. W., Hawkins, E. J., Nielsen, S. L. \& Goates, M. (2002). Enhancing psychotherapy outcomes via providing feedback on client progress: A replication. Clinical Psychology \& Psychotherapy, 9, 91-103.

Landsverk, J., Brown, C. H., Reutz, J. R., Palinkas, L., \& Horwitz, S. M. (2011). Design elements in implementation research: A structured review of child welfare and child mental health studies. Administration and Policy in Mental Health and Mental Health Services Research, 38, 54-63.

Lewis, B. L., Hatcher, R. L., \& Pate II, W. E. (2005). The practicum experience: A survey of practicum site coordinators. Professional Psychology: Research and Practice, 36, 291-298. doi: 10.1037/0735-7028.36.3.291

Manuel, J. K., Hagedorn, H. J., \& Finney, J. W. (2011). Implementing evidence-based psychosocial treatment in specialty substance use disorder care. Psychology of Addictive Behaviors, 25, 225-237. doi:10.1037/a0022398

Margison, F., Barkham, M., Evans, C., McGrath, G., Mellor-Clark, J., Audin, K., \& Connell, J. (2000). Measurement and psychotherapy: Evidence-based practice and practice-based evidence. British Journal of Psychiatry, 177, 123-130. 
McGrew, J. H., Bond, G. R., Dietzen, L., \& Salyers, M. (1994). Measuring the fidelity of implementation of a mental health program model. Journal of Consulting and Clinical Psychology, 62, 670-678. doi: 10.1037/0022-006X.62.4.670

McHugh, R. K., \& Barlow, D. H. (2010). The dissemination and implementation of evidencebased psychological treatments: A review of current efforts. American Psychologist, 73, 7384.

McLean, C. P., \& Foa, E. B. (2011). Prolonged exposure therapy for post-traumatic stress disorder: A review of evidence and dissemination. Expert Reviews, 11, 1151-1163.

McLeod, B. D., \& Weisz, J. R. (2005). The Therapy Process Observational Coding SystemAlliance scale: Measure characteristics and prediction of outcome in usual clinical practice. Journal of Consulting and Clinical Psychology, 73, 323-333.

McLeod, B. D., \& Weisz, J. R. (2010). Therapy process observational coding system for child psychotherapy strategies scale. Journal of Clinical Child and Adolescent Psychology, 39, 436-443.

McMahon, S. D., Ribordy, S. C., \& Washburn, J. J. (2002). Training clinics and community outreach: Integration of service, training, and research. The Behavior Therapist, 25, 122-127.

Merry, S. N., Stasiak, K., Shepherd, M. Frampton, C., Fleming, T., \& Lucassen, M. F. G. (2012). The effectiveness of SPARX, a computerized self help intervention for adolescents seeking help for depression: Randomised controlled non-inferiority trial. British Medical Journal, 344:e2598. doi: 10.1136/bmj.e2598

Morland, L. A., Greene, C. J., Rosen, C. S., Foy, D., Reilly, P., Shore, J., . . Frueh, B. C. (2010). Telemedicine for anger management therapy in a rural population of combat veterans with posttraumatic stress disorder: A randomized noninferiority trial. Journal of Clinical 
Psychiatry, 71, 855-863. doi:10.4088/JCP.09m05604blu

Miller, W. R., Yahne, C. E., Moters, T. B., Martinez, J. \& Pirritano, M. (2004). A randomized trial of methods to help clinicians learn motivational interviewing. Journal of Consulting and Clinical Psychology, 72, 1050-1062.

NAMI (2003). An update on evidence-based practices in children's mental health. Retrieved from:http://www.nami.org/Template.cfm?Section=Child_and_Adolescent_Action_Center\&T emplate=/ContentManagement/ContentDisplay.cfm\&ContentID=12717

Nathan, P. E., \& Gorman, J. M. (Eds.). A guide to treatments that work, $3^{\text {rd }}$ edition. Oxford, UK: Oxford University Press.

NIMH. (2009). Dissemination and implementation research in health (R21). Retrieved March 14, 2013, from http://grants.nih.gov/grants/guide/pa-files/par-10-040.html

The National Advisory Mental Health Council Workgroup on Child and Adolescent Mental Health Intervention and Deployment. (2001). Blueprint for change: Research on child and adolescent mental health. Washington, D.C. Department of Health and Human Services, Public Health National Institutes of Health.

Palinkas, L. A., Aarons, G. A., Horwitz, S., Chamberlain, P., Hurlburt, M., \& Landsverk, J. (2011). Mixed method designs in implementation research. [Research Support, N.I.H., Extramural Review]. Administration and Policy in Mental Health and Mental Health Services Research, 38, 44-53. doi: 10.1007/s10488-010-0314-z

Palinkas, L. A., Schoenwald, S. K., Hoagwood, K., Landsverk, J., Chorpita, B. F., Weisz, J. R., \& Health, t. R. N. o. Y. M. (2008). An ethnographic study of implementation of evidencebased treatments in child mental health: First Steps. Psychiatric Services, 59, 738-746.

Pelham, W. E., Gnagy, E. M., Greiner, A. R., Waschbusch, D. A., Fabiano, G. A., \& Burrows- 
MacLean, L. (2010). Summer treatment programs for attention-deficit/hyperactivity disorder. In J. R. Weisz \& A. E. Kazdin (Eds.), Evidence-based psychotherapies for children and adolescents (2nd ed., pp. 277-292). New York, NY: Guilford Press.

Perepletchikova, F., Treat, T. A., \& Kazdin, A. E. (2007). Treatment integrity in psychotherapy research: Analysis of the studies and examination of the associated factors. Journal of Consulting and Clinical Psychology, 75, 829-841.

Raine, R., Sanderson, C., Hutchings, A., Carter, S., Larkin, K., \& Black, N. (2004). An experimental study of determinants of group judgments in clinical guideline development. Lancet, 364, 429- 437.

Rotheram-Borus, M. J., Swendeman, D., \& Chorpita, B. F. (2012). Disruptive innovations for designing and diffusing evidence-based interventions. American Psychologist, 67, 463-476. doi: $10.1037 / \mathrm{a} 0028180$

Ruzek, J. I., Karlin, B. E., \& Zeiss, A. (2012). Implementation of evidence-based psychological treatments in the Veterans Health Administration. In R. K. McHugh \& D. H. Barlow (Eds.), Dissemination and implementation of evidence-based psychological interventions (pp. 78-96). New York, NY: Oxford University Press.

Safran, J. D., Abreu, I., Oglive, J., \& DeMaria, A. (2011). Does psychotherapy research influence the clinical practice of researcher-clinicians? Clinical Psychology Science and Practice, 18, 357-371.

Saldana, L., \& Chamberlain, P. (2012). Supporting implementation: The role of community development teams to build infrastructure. American Journal of Community Psychology, 50, 334-346. doi: 10.1007/s10464-012-9503-0

Sanders, M. R. (2012). Development, evaluation, and multinational dissemination of the Triple P 
- Positive Parenting Program. Annual Review of Clinical Psychology, 8, 345-379.

Schoenwald, S. K. (2010). From policy pinball to purposeful partnership: The policy contexts of multisystemic therapy transport and dissemination. In J. R. Weisz \& A. E. Kazdin (Eds.), Evidence-based psychotherapies for children and adolescents (pp. 538-556). New York: Guilford Press.

Scott, S. (2010). National dissemination of effective parenting programmes to improve child outcomes. British Journal of Psychiatry, 196, 1-3.

Sharkin, B. S., \& Plageman, P. M. (2003). What do psychologists think about mandatory continuing education? A survey of Pennsylvania practitioners. Professional Psychology: Research and Practice, 34, 318-323. doi:10.1037/0735-7028.34.3.318

Shoham, V., \& Insel, T. R. (2011). Rebooting for whom? Portfolios, technology, and personalized intervention. Perspectives on Psychological Science, 6, 478-482.

Sholomskas, D. E., Syracuse-Siewart, G., Rounsaville, B. J., Ball, S. A., Nuro, K. F., \& Carroll, K. M. (2005). We don't train in vain: A dissemination trial of three strategies of training clinicians in cognitive-behavioral therapy. Journal of Consulting and Clinical Psychology, 73, 106-115.

Silverman, W. K,, \& Hinshaw, S. P. (2008). Evidence-based psychosocial treatments for children and adolescents: A ten-year update. Journal of Clinical Child and Adolescent Psychology, 37, 1-7. doi: 10.1080/15374410701817725

Smith, J. L., Carpenter, K. M., Amrhein, P. C., Brooks, A. C., Levin, D., Schreiber, E. A., .. . Nunes, E. V. (2012). Training substance abuse clinicians in motivational interviewing using live supervision via teleconferencing. Journal of Consulting and Clinical Psychology, 80, 450-464. doi: 10.1037/a0028176 
Stein, B. D., Kogan, J. N., Hutchison, S. L., Magee, E. A., \& Sorbero, M. J. (2010). Use of outcomes information in child mental health treatment: Results from a pilot study. Psychiatric Services, 61, 1211-1216.

Stewart, R. E., \& Chambless, D. L. (2007). Does psychotherapy research inform treatment decisions in private practice? Journal of Clinical Psychology, 63, 267-281. doi: 10.1002/jclp.20347

Stewart, R. E., Stirman, S. W., \& Chambless, D. L. (2012). A qualitative investigation of practicing psychologists' attitudes toward research-informed practice: Implications for dissemination strategies. Professional Psychology: Research and Practice, 43, 100-109. doi: 10.1037/a0025694.supp

Stice, E., Burton, E., Bearman, S. K., \& Rohde, P. (2006). Randomized trial of a brief depression prevention program: An elusive search for a psychosocial placebo control condition. Behaviour Research and Therapy, 45, 863-876. doi: 10.1016/j.brat.2006.08.008

Stirman, S. W., Kimberly, J., Cook, N., Calloway, A., Castro, F., \& Charns, M. (2012). The sustainability of new programs and innovations: A review of the empirical literature and recommendations for future research. Implementation Science, 7: 17. doi:10.1186/17485908-7-17

Straus, S. E., Richardson, W. S., Glasziou, P., \& Haynes, R. B. (2005). Evidence-based medicine: How to practice and teach EBM (3rd ed.). London: Churchill Livingstone.

VandeCreek, L., Knapp, S., \& Brace, K. (1990). Mandatory continuing education for licensed psychologists: Its rationale and current implementation. Professional Psychology: Research and Practice, 21, 135-140.

Wampold, B. E., Budge, S. L., Laska, K. M., Del Re, A. C., Baardseth, T. P., .. . Gunn, W. 
(2011). Evidence-based treatments for depression and anxiety versus treatment-as-usual: A met-analysis of direct comparisons. Clinical Psychology Review, 31, 1304-1312. doi: 10.1016/j.cpr.2011.07.012

Webster-Stratton, C., \& Reid, M. J. (2010). Treating conduct problems in young children using The Incredible Years Parents, Teachers, and Children training series. In J. R. Weisz \& A. E. Kazdin (Eds.), Evidence-based psychotherapies for children and adolescents (2nd ed., pp. 194-210). New York, NY: Guilford Press.

Weissman, M. M., Verdeli, H., Gameroff, M. J., Bledsoe, S. E., Betts, K., Mufson, L., .. . Wickramaratne, P. (2006). National Survey of Psychotherapy Training in Psychiatry, Psychology, and Social Work. Archives of General Psychiatry, 63, 925-934. doi:

10.1001/archpsyc.63.8.925

Weisz, J. R. (2004). Psychotherapy for children and adolescents: Evidence-based treatments and case examples. New York: Cambridge University Press.

Weisz, J. R., Chorpita, B. F., Frye, A., Ng, M. Y., Lau, N., Bearman, S. K., . . . the Research Network on Youth Mental Health (2011). Youth top problems: Using idiographic, consumerguided assessment to identify treatment needs and track change during psychotherapy. Journal of Consulting and Clinical Psychology, 79, 369-380.

Weisz, J. R., Chorpita, B. F., Palinkas, L., Schoenwald, S. K., Miranda, J., Bearman, S. K., . . . the Research Network on Youth Mental Health. (2012). Testing standard and modular designs for psychotherapy treating depression, anxiety, and conduct problems in youth: A randomized effectiveness trial. Archives of General Psychiatry, 69, 274-282.

Weisz, J. R., \& Gray, J. S. (2008). Evidence-based psychotherapies for children and adolescents: Data from the present and a model for the future. Child and Adolescent Mental Health, 13, 
54-65.

Weisz, J. R., Jensen-Doss, A. J., \& Hawley, K. M. (2005). Youth psychotherapy outcome research: A review and critique of the evidence base. Annual Review of Psychology, 56, 337363.

Weisz, J. R., Jensen-Doss, A., \& Hawley, K. M. (2006). Evidence-based youth psychotherapies versus usual clinical care: A meta-analysis of direct comparisons. American Psychologist, 61, $671-689$.

Weisz, J. R., \& Kazdin, A. E. (2010). Evidence-based psychotherapies for children and adolescents. New York: Guilford Press.

Weisz, J. R., Kuppens, S., Eckshtain, D., Ugueto, A. M., Hawley, K. M., \& Jensen-Doss, A. (in press). Do evidence-based youth psychotherapies outperform usual clinical care? A multilevel meta-analysis. JAMA Psychiatry.

Weisz, J. R., Southam-Gerow, M. A., Gordis, E. B., Connor-Smith, J. K., Chu, B. C., Langer, D. A., ... Weiss, B. (2009). Cognitive-behavioral therapy versus usual clinical care for youth depression: An initial test of transportability to community clinics and clinicians. Journal of Consulting and Clinical Psychology, 77, 383-396.

Weisz, J. R., Uqueto, A. M., Cheron, D. M., \& Herren, J. (2013). Evidence-based youth psychotherapy in the mental health ecosystem. Journal of Clinical Child and Adolescent Psychology, 42, 274-286. doi: 10.1080/15374416.2013.764824

Woody, S. R., Weisz, J, \& McLean, C. (2005). Empirically supported treatments: 10 years later. The Clinical Psychologist, 58(4), 5-11.

Yates, B. T. (2011). Delivery systems can determine therapy costs and effectiveness, more than type of therapy. Perspectives on Psychological Science, 6, 498-502. 


\section{Author Notes}

Some of the research reported here was supported by grants from the National Institute of Mental Health (MH57347, MH068806, MH085963), the Norlien Foundation, the MacArthur Foundation, and the Annie E. Casey Foundation. We are grateful Katherine Corteselli, Lea Petrovic for their valuable help with this manuscript, and to the children, families, clinicians, clinic administrators, and government program and policy leaders who have participated in and supported our research and enriched our thinking. John Weisz receives royalties for some of the published works cited in this paper. Otherwise, none of the authors reports any potential conflict of interest. Address correspondence about this article to John R. Weisz, Department of Psychology, Harvard University, William James Hall, 33 Kirkland Street, Cambridge, MA 02138. 


\section{Table 1}

Percentage of Groups in Youth Psychotherapy Outcome Studies that Employed Clinically Representative Youths, Therapists, and Treatment Settings

\begin{tabular}{|c|c|c|c|c|c|c|}
\hline Decade & $1960 s$ & $1970 s$ & $1980 \mathrm{~s}$ & $1990 \mathrm{~s}$ & $2000 s$ & All \\
\hline No. of Studies & 13 & 62 & 99 & 100 & 187 & 461 \\
\hline No. of Groups & 35 & 183 & 273 & 244 & 425 & 1160 \\
\hline \multicolumn{7}{|l|}{ How Youths Were Enrolled in Study } \\
\hline Recruited, non-treatment-seeking & 62.9 & 85.8 & 65.9 & 57.8 & 62.8 & 66.1 \\
\hline Clinic-referred, treatment-seeking & 5.7 & 4.9 & 24.2 & 26.2 & 24.5 & 21.1 \\
\hline Required via court/justice system & 17.1 & 8.2 & 8.8 & 11.5 & 7.8 & 9.1 \\
\hline Enrollment method not reported & 14.3 & 1.1 & 1.1 & 4.5 & 4.9 & 3.6 \\
\hline \multicolumn{7}{|l|}{ Who Provided the Treatment } \\
\hline$\leq 50 \%$ therapists are practitioners & 65.7 & 42.1 & 55.3 & 40.2 & 35.5 & 43.1 \\
\hline$>50 \%$ therapists are practitioners & 2.9 & 9.8 & 7.7 & 10.7 & 19.1 & 12.7 \\
\hline Therapist vocation not reported & 14.3 & 27.9 & 23.4 & 32.0 & 25.4 & 26.4 \\
\hline No treatment/waitlist & 17.1 & 20.2 & 13.6 & 17.2 & 20.0 & 17.8 \\
\hline
\end{tabular}

Where Treatment Took Place

$\begin{array}{lcccccc}\text { Research setting } & 11.4 & 6.6 & 9.9 & 18.4 & 19.5 & 14.7 \\ \text { Custodial, school, supervised setting } & 8.6 & 29.5 & 32.6 & 20.5 & 22.6 & 25.2 \\ \text { Clinical service setting } & 0 & 2.7 & 5.9 & 11.5 & 14.8 & 9.7\end{array}$




$\begin{array}{lcccccc}\text { Correctional setting } & 14.3 & 1.1 & 5.1 & 4.1 & 2.1 & 3.4 \\ \text { Treatment setting not reported } & 48.6 & 39.9 & 33.0 & 28.3 & 20.9 & 29.1 \\ \text { No treatment/waitlist } & 17.1 & 20.2 & 13.6 & 17.2 & 20.0 & 17.8\end{array}$

Sum of Representativeness Factors

$\begin{array}{lcccccc}\text { No factors reported } & 91.4 & 83.6 & 70.0 & 63.9 & 61.2 & 68.3 \\ \text { One factor reported } & 8.6 & 15.3 & 22.7 & 25.4 & 23.8 & 22.1 \\ \text { Two factors reported } & 0 & 1.1 & 7.0 & 9.0 & 10.6 & 7.6 \\ \text { Three factors reported } & 0 & 0 & 0.4 & 1.6 & 4.5 & 2.1\end{array}$

Note. Because treatment provider and setting are group-level variables, percentages of groups rather than percentages of studies are reported for all three variables. Three studies that employed a combination of non-treatment seeking and treatment seeking youths, and one study with a treatment condition that employed a combination of clinical and research settings, were excluded from analyses. [Reprinted with permission, John R. Weisz] 


\section{Table 2}

\section{Web-based Resources for Dissemination and Implementation of Evidence-based Practices}

\section{Organization and Homepage Key Features}

Division 12 (Society of Clinical Psychology), American

Psychological Association (APA)

http://psychologicaltreatments.org/

Division 53 (Society of Clinical Child and Adolescent

Psychology), American Psychological Association

(APA), and Association of Behavioral and

Cognitive Therapies (ABCT)

http://effectivechildtherapy.com/

National Registry of Evidence-based Programs and

Practices (NREPP), Substance Abuse and Mental

Health Services Administration (SAMHSA), US

Department of Health and Human Services.

http://www.nrepp.samhsa.gov/

Blueprints for Healthy Youth Development (Blueprints), Center for the Study and Prevention of Violence (CSPV), University of Colorado Boulder http://www.blueprintsprograms.com/
List and description of psychotherapies for adult

psychological disorders designated as having Strong, Modest, No, or Controversial Research Support; key research references; information on or links to treatment manuals, centers/workshops providing training opportunities, training DVDs, and developers List and description of psychotherapies for youth psychological disorders designated as Well-Established, Probably Efficacious, Possibly Efficacious, or Experimental; key research references; links to treatment manuals, self-help materials, relevant articles, developer/publisher websites

Searchable online database of programs designed to prevent or treat psychological disorders or to promote mental health; numerical ratings for research quality and dissemination readiness; summaries of evidence; information on implementation history, costs, contacting developer

Searchable online database of programs targeting youth psychological and physical health, education, and positive relationships; designates programs as Model or Promising based on research quality, dissemination readiness, and intervention impact and specificity; summaries of evidence; information on training and assistance by 
What Works Clearinghouse (WWC), Institute of

Education Sciences (IES), US Department of

Education

http://ies.ed.gov/ncee/wwc/

The Cochrane Collaboration, international network of researchers, multiple centers and branches worldwide, secretariat is based in Oxford, England http://www.cochrane.org/

The Campbell Collaboration, international network of researchers, secretariat hosted by the Norwegian Knowledge Center for the Health Services http://www.campbellcollaboration.org/

National Implementation Research Network (NIRN), Frank Porter Graham Child Development Institute, University of North Carolina at Chapel Hill http://nirn.fpg.unc.edu/

Community Engagement Program, Clinical Translational Science Institute (CTSI), University of California, San Francisco http://accelerate.ucsf.edu/research/community developer, costs, and funding strategies

Systematic reviews of interventions in education, including school-based, school staff-delivered, or school-directed psychotherapies for “Children Classified as Having an Emotional Disturbance” or that support "Early Childhood Education for Children with Disabilities”

Systematic reviews and meta-analyses of healthcare practices and research methodology, including psychotherapies; some reviews include cost-effectiveness outcomes

Systematic reviews and meta-analyses of interventions in the areas of crime and justice, education, international development, and social welfare

Descriptions of implementation concepts and models, recommendations implementation science and practice, and sample materials used in the actual implementation of several interventions

Downloadable manuals for researchers, community organizations, and community clinicians and FAQ pages on how to participate in community-engaged research, including implementing an intervention and evaluating intervention and implementation outcomes 


\section{Table 3}

Agenda for the Future: Directions for Practice, Research, and Training

\section{Making Intervention Research Look More Like Practice}

Building the knowledge base needed for dissemination and implementation by making studies more clinically representative.

\section{Solving the “Too Many EBPs" Problem}

Applying more rigorous standards to determine which “evidence-based practices” will improve on options currently available, and thus warrant implementation.

\section{Addressing the Mismatch between Interventions and Users}

Designing interventions to address comorbidity, flux, diverse caseloads, and other characteristics of the clients and therapists who use them.

\section{Building Efficient and Accessible Delivery Methods and Models}

Expanding population impact by using self-help books, internet and computer technology, videoconferencing, paraprofessional outreach, and other complements to individual sessions with a professional therapist.

\section{Solving the “How'm I Doing?" Problem}

Building systems to monitor client response to treatment and providing ongoing feedback to clinicians, to guide decision-making throughout episodes of care.

\section{Embracing High-Risk/High-Gain Innovation and Learning from Failure}

Recognizing and incentivizing research on innovative implementation strategies that may fail; addressing the training and career challenges associated with high-risk/high-gain approaches.

\section{Contemplating New Rules and Professional Tracks}


Thinking through new rules, ethical principles, and professional tracks (e.g., "trialists,” “evidence brokers”) that may be needed in the increasingly entrepreneurial and proprietary world of clinical psychological science, as conflict-of-interest concerns expand.

\section{Deciding Who Owns the Cure}

Launching a discussion about the intellectual property (IP) associated with successful interventions, focusing in part on which ownership arrangements will best serve those who need effective mental health care.

\section{Building Community-Research Partnerships}

Giving priority_-in research and training — to the creation of the community-research partnerships that will be essential in studies of EBP dissemination and implementation.

\section{Courtship and Marriage of DIP and DIS}

Finding ways to link and synchronize the often-insular practice and science of DI-for example, by ensuring that every demonstration project is designed to answer important scientific questions about dissemination-implementation. 


\section{Figure Caption}

Figure 1. Effect sizes of individual studies comparing evidence-based youth psychotherapies (EBPs) to usual care. Horizontal bar at .29 shows mean effect size across the full study set. Note the number of studies for which usual care showed effects similar to or superior to EBPs. [Reprinted with permission, John R. Weisz] 


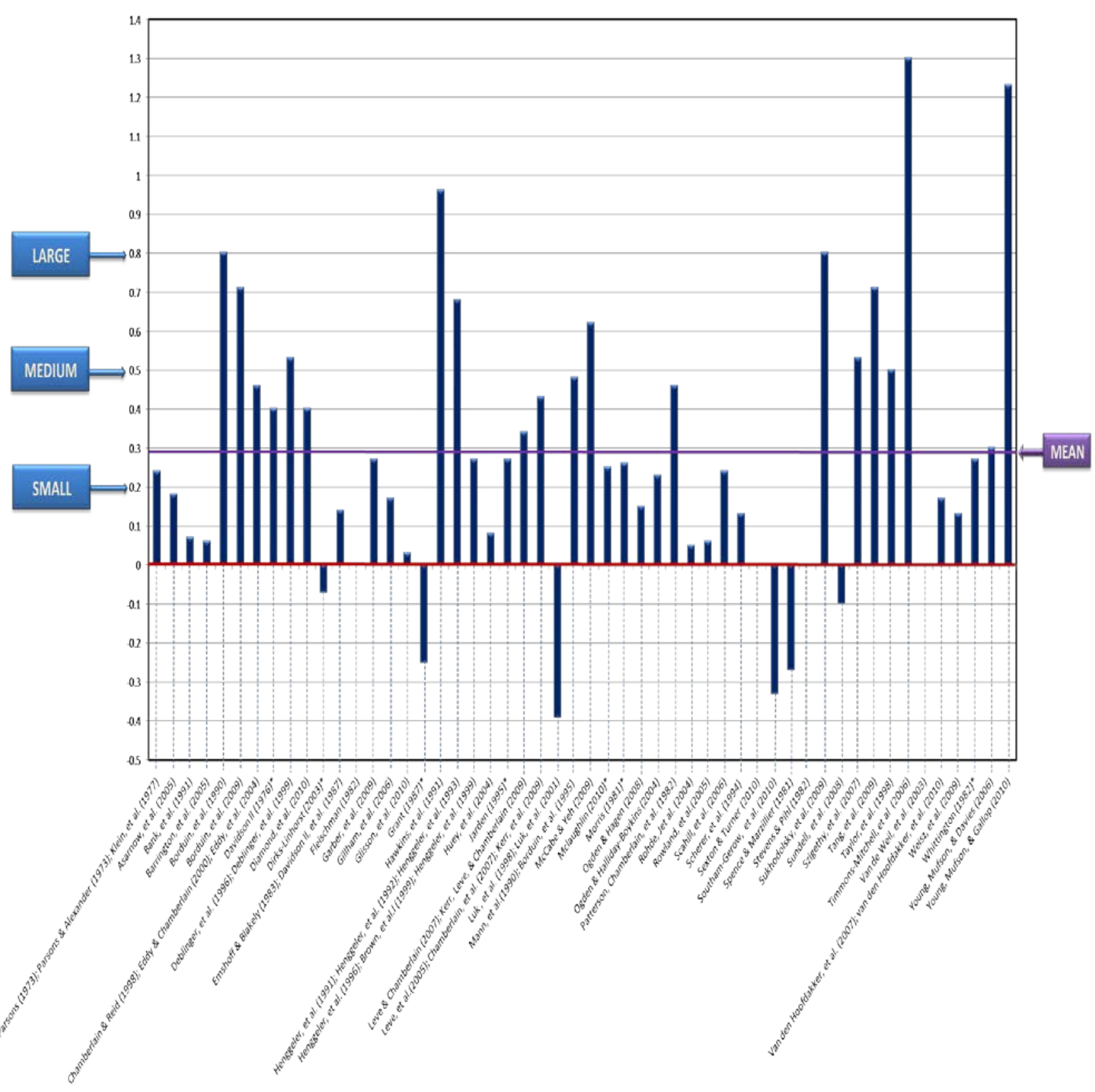

